\section{The Effect of Modified PVA Interfacial Layer Doped by Zn Nanoparticles on the Electrical Parameters of Au/N-4H Sic (MS) Structures}

\section{Abstract}

Both $\mathrm{Au} / \mathrm{n}-4 \mathrm{H}$ SiC (MS) and Au/(Zn doped-PVA)/n-4H SiC (MPS) structures were fabricated by using the same wafer. This effect of modified PVA interlayer doped by $\mathrm{Zn}$ nanoparticles on main electrical parameters, such as ideality factor $(n)$, barrier height $(\mathrm{BH})$, series resistance $\left(R_{s}\right)$, and rectification rate $(R R)$ were evaluated and investigated by using the current-voltage $(I-V)$ data measurements. Furthermore, the energy dependent profile of surface states $\left(N_{s s}\right)$ was also obtained from these data by taking into account voltage dependent $\mathrm{BH}$ and $\mathrm{n}$. The sources of observed negative capacitance (NC) at the accumulation region for the MS and MPS structures were also evaluated. From capacitance-voltage $(C-V)$ and conductancevoltage $(G / \omega-V)$ data measurements, the profile of $R_{s}$ and $N_{s s}$ were also evaluated and investigated as voltage dependent at high frequency ( $1 \mathrm{MHz}$ ) by using Nicollian-Brews technique. These results explain how is the effect of modified PVA interfacial layer doped by $\mathrm{Zn}$ nanoparticles on increasing in the RR and decreasing of the values of $R_{s}, N_{s s}$, and leakage current of MPS structure as compared with MS structure. Thus, we can say that this increase in the performance of the MPS structure is a result of the grown of the (Zn-doped PVA) layer between Au and $\mathrm{n}-4 \mathrm{H} \mathrm{SiC}$.

Keywords: MS and MPS structures; Passivation effect; Surface states; Negative capacitance

\section{Al-Dharob $\mathrm{MH}^{1}{ }^{,}$Kökce $\mathrm{A}^{1 *}$, Lapa HE ${ }^{1}$, Özdemir AF ${ }^{1}$ and Altındal S ${ }^{2}$}

1 Department of Physics, Faculty of Arts and Sciences, Süleyman Demirel University, Isparta, Turkey

2 Department of Physics, Faculty of Sciences, Gazi University, Ankara, Turkey

\section{*Corresponding author: Kökce A \\ alikokce@sdu.edu.tr \\ Department of Physics, Faculty of Arts and Sciences, Süleyman Demirel University, Isparta, Turkey.}

Tel: 2462114031

Citation: Al-Dharob MH, Kökce A, Lapa HE, Özdemir AF, Altindal S (2018) The Effect of Modified PVA Interfacial Layer Doped by Zn Nanoparticles on the Electrical Parameters of $\mathrm{Au} / \mathrm{N}-4 \mathrm{H}$ Sic (MS) Structures. Polym Sci Vol.4 No.1:2

Received: September 19, 2017; Accepted: November 28, 2017; Published: March 10, 2018

\section{Introduction}

Recently, many of experimental researches and studies for the metal-polymer-semiconductor (MPS) type structures in order to replacing the traditional metal-semiconductor (MS) structures with and without insulation interfacial layer such as $\mathrm{SiO}_{2}$ or $\mathrm{SnO}_{2}$ [1-7]. In general, pure polymer usually is a poor conductivity material, but its conductivity can be increased by using doping with a metal at appropriate rates [8-11]. Therefore, polymer interfacial layers attract considerable attention in the electronic and optoelectronic devices due to their easy device fabrication, flexibility, low cost, suitability.

There are different kinds of polymers/organics among of them is poly (vinyl alcohol) (PVA) that has a semi-crystalline structure and high water-solubility, nontoxic, crystallinity with wide range, good charge/energy storage capacity and high dielectric strength properties [12-15].
In addition, PVA is from hydroxyl group; therefore it can be reacts easily with other organic and inorganic compounds. Therefore, in very recently, it is believed that MPS structure is considered one of the most promising candidates in the future semiconductor technology instead of classic/conventional MS, MOS and MIS type structures [8-15].

At the same time, silicon carbide $(\mathrm{SiC})$ represents a very important semiconductor among many kinds of semiconductor crystals with wide band-gap $\left(E_{g}\right)$ [16]. Furthermore, $\mathrm{SiC}$ is 3 times wider $E_{g}$ $(=3.2 \mathrm{eV})$ and 10 times higher critical electric field $\left(E=2.2 \times 10^{6} \mathrm{~V} /\right.$ $\mathrm{cm}$ ) when compared conventional Si semiconductor [17].

It is clear that the electrical properties of MS structure with exist of insulator or organic interfacial layer and without it usually not only depend on the metal and interfacial layer used, but depend strongly on the surface properties, doping concentration level of semiconductor, barrier form at the interface between metal 
and semiconductor, series and shunt resistances of the device $\left(R_{s}\right.$ and $\left.R_{s h}\right)$ and applied bias voltage across the junction [1826]. Especially, high-dielectric interlayer can cause the leakage reverse current, $n, R_{s}$ and $N_{s s}$ and increase the $\mathrm{BH}$, intercept voltage $\left(V_{i}\right.$ or $\left.V_{o}\right)$ and $R_{s h}$ [27]. Zinc (Zn), Cobalt (Co) and nickel (Ni) were used as doped materials due to low cost, abundant raw material and heat cycling. The other more important feature of the $\mathrm{SiC}$ is the ability to form $\mathrm{SiO}_{2}$ as a native insulator/oxide layer. There are still a number of factors which are limiting the device performance such as the formation of back Ohmic contact with low resistivity and high-temperature stable Schottky/rectifier contacts. Both $\mathrm{Zn}$ and $\mathrm{SiC}$ have also the same wurtzite crystal symmetry and relatively higher lattice mismatch (5\%). Although there are various "poly-types" SiC, but the most preferred among is $4 \mathrm{H}-\mathrm{SiC}$ due to its superior physical properties through owing double electron mobility as compare with $6 \mathrm{H}-\mathrm{SiC}$ at a given dopant density and the availability of high-quality epitaxial wafers [27-30].

In this study, the experimental result give us a good indicate about how is the effect of modified PVA interfacial layer doped by $\mathrm{Zn}$ nanoparticles on electrical properties and conduction/transport mechanism, therefore, after we fabricate $\mathrm{Au} / \mathrm{n}$-SiC (MS) and Au/ (Zn-PVA)/n-4H SiC (MPS) structures, we calculate and investigate the main electrical parameters for both structures to compare between them by using the forward and reverse bias I-V, $C-V$ and $G / \omega-V$ measurements at room temperature. The interface state energy distribution profiles of $N_{s s}$ were obtained from the forward bias $I-V$ data for two type structures by taking into account voltage dependent $\mathrm{BH}$ and ideality factor. As a results of comparison between the two structures, it was found that the modified PVA interfacial layer doped by Zn nanoparticles leads to a decrease in the values $N_{s s}, R_{s}$, leakage current and increase in shunt resistance $\left(R_{s h}\right)$ and $R R$.

\section{Experimental Details}

$\mathrm{Au} / \mathrm{n}-4 \mathrm{H}$ SiC (MS) and Au/Zn-PVA/n-SiC (MPS) with modified PVA interfacial layer doped by $\mathrm{Zn}$ nanoparticles structures were fabricated by using two same quarter n-type $4 \mathrm{H}-\mathrm{SiC}$ (0001) wafer with the following properties: donor concentration atoms $\left(\mathrm{N}_{\mathrm{d}}\right)$ about $7.07 \times 10^{17} \mathrm{~cm}^{-3}$, rectifying contact area $0.0314 \mathrm{~cm}^{2}$ and thickness about $250 \mu \mathrm{m}$. for fabrication process, firstly, n-type $4 \mathrm{H}-\mathrm{SiC}$ wafer was dipped in organic solution of ammonium peroxide solution for 10 minutes to remove any oxide thin layer that could be cover it, and then etched in a sequence of acidic solution $\mathrm{H}_{2} \mathrm{O}+\mathrm{HCl}$ by using ultrasonic bath and then quenched in de-ionized water (DI) with $18 \mathrm{M} \Omega . \mathrm{cm}$ resistivity for 10 minutes. Preceding each cleaning step, the wafer was rinsed thoroughly in $\mathrm{DI}$ water. Finally, $\mathrm{n}-4 \mathrm{H} \mathrm{SiC}$ wafer dried in the high-pure dry nitrogen $\left(\mathrm{N}_{2}\right)$ gas to prevent any oxidation of the wafer and then high purity (99.999\%) Au with $1500 \AA$ thickness was thermally evaporated onto the whole back side of $\mathrm{n}$ type $4 \mathrm{H}-\mathrm{SiC}$ wafer at $10^{-6}$ Torr in the metal evaporation system (MES). In order to perform a low resistivity Ohmic back contact, $\mathrm{n}-4 \mathrm{H}$ SiC wafer was sintered at $500^{\circ} \mathrm{C}$ for 5 minutes in the nitrogen ambient.

After the formation of ohmic contact, firstly, high purity Au dots with $0.0314 \mathrm{~cm}^{2}$ and $1500 \AA$ thickness was thermally evaporated onto the front of the first quarter $\mathrm{n}-4 \mathrm{H}$ SiC wafer in the same MES. By this way the fabrication processes of $\mathrm{Au} / \mathrm{n}-4 \mathrm{H} \mathrm{SiC}$ (MS) were completed. Secondly, the prepared (Zn-doped PVA) solution was deposited onto the front of the second quarter $n-4 \mathrm{H}$ SiC wafer by using electrospinning method. Lastly, like the first sample high purity Au dots with $0.0314 \mathrm{~cm}^{2}$ and $1500 \AA$ thickness was also thermally evaporated onto the front of the second quarter and so on the fabrication processes of Au/Zn-PVA/nSiC (MPS) structures were also completed. The metal thickness layer and the deposition rates were monitored with the help of quartz crystal thickness monitor. The schematic diagrams of the fabricated of MS and MPS type structures were given in Figure 1a and $\mathbf{1 b}$, respectively.

In order to obtain the $I-V, C-V$ and $G /-V$ measurements for both MS and MPS structures, they were mounted on a copper $(\mathrm{Cu})$ holder with the help of silver paste and electrical measurements were made to the upper electrodes by the use of tiny silver coated Cu-wires with silver paste. The measurement system was also given in Figure 2. The thickness of (Zn-PVA) $\left(d_{i}\right)$ was determined as $50 \mathrm{~nm}$ from the high frequency $(1 \mathrm{MHz}) \mathrm{C}-\mathrm{V}$ plot of the interlayer capacitance $\left(C_{i}=\varepsilon_{\text {ro }} A / d_{i}\right)$ at the strong accumulation region. As shown in Figure 2, the $C-V$ and $G / \omega-V$ measurements were performed by the use of a computerized HP 4192A LF impedance analyzer (5 Hz-13 MHz) at low (1 kHz) and high (1 $\mathrm{MHz}$ ) frequencies. On the other hand, the I- $\mathrm{V}$ measurements were performed by the use of a Keithley 2400 I-V source-meter. In addition, all measurements were carried out in dark with the help of a microcomputer through an IEEE-488 AC/DC converter card in the JANES-475 Cryostat at about $10^{-3} \mathrm{Torr}$ to avoid an external noise or other environmental effects (Figure 2).

\section{Results and Discussion}

\section{The forward and reverse bias current-voltage (I-V) characteristics}

In order to determine whether or not a MS structure with modified PVA interfacial layer doped by Zn nanoparticles and without an interfacial layer has the ideal diode behavior, its experimental results at forward bias $I-V$ characteristics and low or intermediate voltage $(V 3 k T / q)$ can be analyzed according to thermionic emission (TE) theory [18-20].

$$
I=\underbrace{A A^{*} T^{2} \exp \left(-\frac{q \hat{\mathrm{O}}_{B 0}}{k T}\right)}_{I}\left[\exp \left(\frac{q V_{D}}{n k T}\right)-1\right]
$$

The pre-factor or the first part of the eqn. (1) represent the saturation current $\left(I_{0}\right)$ calculated from straight line intercept of $\mathrm{Lnl}$ at $\mathrm{V}=0, A^{*}$ is the effective Richardson constant $\left(146 \mathrm{~A} . \mathrm{cm}^{-2} \mathrm{~K}^{-2}\right.$ for n-type $4 \mathrm{H} \mathrm{SiC,} \mathrm{A} \mathrm{is} \mathrm{the} \mathrm{rectifier/Schottky} \mathrm{contact} \mathrm{area,} T$ is the absolute temperature in $K, V_{D}$ is the voltage drop across the junction ad $n$ is the ideality factor [19], and the other quantities are well known in the literature. Figure $\mathbf{3}$ shows the experimental reverse and forward bias $I-V$ characteristics for MS and MPS type structures at room temperature. As shown in Figure 3, both MS and MPS type structures have a rectifying behavior with exponential increase of the current showing in the forward bias and weak voltage dependence in the reverse bias regions. The 
value of $I_{0}$ was evaluated from the intercept of the linear region of $\mathrm{LnI-V}$ plots, whereas the value of $\mathrm{n}$ was evaluated from its slope for both structures according to the following equation $[18,19]$.

$n=q / k T\left(\frac{d V}{d(\ln I)}\right)=1+\frac{\delta_{i}}{\varepsilon_{i}}\left\lceil\frac{\varepsilon_{s}}{W_{D}}+q N_{s s}\right\rceil$

Where, $\delta_{i}$ represent the thickness of interlayer, $W_{D}$ represent the depletion layer width, $N_{s s}$ represent the surface states/traps, $\varepsilon_{i}$ and $\varepsilon_{s}$ represents the permittivity of modified PVA interfacial layer doped by Zn nanoparticles and semiconductor, respectively.

Now, the value of $\Phi_{B o}$ was calculated by using theoretical value of $A^{*}$ and extrapolated $I_{o}$ values as following equation for two type structures [20].

$\hat{O}_{B 0}=\frac{k T}{q} \ln \left(\frac{A A^{*} T^{2}}{I_{0}}\right)$

Figure $3, \mathrm{Ln} /$ vs. $V$ plot of the MPS structure shows two linear regions with different slopes whereas for MS structure this plot has one linear region in the intermediate biases. For the MPS structure, these regions are belonging to low bias voltages $(3 k T / q$ $\leq V \leq 0.55 \mathrm{~V})$ and intermediate bias voltage regions $(0.55 \mathrm{~V} \leq \mathrm{V} \leq$ $1.30 \mathrm{~V})$, respectively. Such two linear regions for MPS structure are known two parallel diodes model with different $\mathrm{BH}$ [31]. In addition, in the enough high forward-bias voltages, Figure 3 plot shows for both MS and MPS structure deviation from linearity due to the existence of $R_{s}$. Table 1 , shows the values of $I_{0}, \mathrm{n}$ and $\Phi_{\mathrm{BO}}$ for MS and MPS structures were found from the first region as $1.90 \times 10^{-11} \mathrm{~A}, 3.854,0.906 \mathrm{eV}$ and $7.08 \times 10^{-10} \mathrm{~A}, 3.24,0.80 \mathrm{eV}$, respectively. These high values of $n$ for two structures at room temperature can especially be due to the existence of native or deposited interlayer, the spatially distribution of $N_{s s}$ and the exist of many distribution of low $\mathrm{BH}$ or patches an average $\mathrm{BH}$ $[8,14,18-20]$. These results refer to the values of $n$ and leakage current at $(-6 \mathrm{~V})$ for the MPS with (Zn doped-PVA) interfacial layer are lower than those of the MS attributed to the passivity role of Zn-PVA interfacial polymer layer. Similarly, the rectifying rate $\left(R=I_{F} / I_{R}\right)$ at $\pm 6 \mathrm{~V}$ for the MPS structure is 15.5 times higher than MS structure (Figure 3 and Table 1).

As can be seen in eqn. (2), for MPS type structure or diode has $N_{s s}$ in equilibrium state with semiconductor $\left(d_{i} \geq 3 \mathrm{~nm}\right)$ and interfacial layer, the experimental results value of $n$ becomes greater than 1 and dependent on applied bias voltage. Thus, $N_{s s}$ can be calculated from the forward bias I-V characteristics by taking into account voltage dependent effective $\mathrm{BH}\left(\Phi_{e}\right)$ and ideality factor $n\left(V_{i}\right)$ by using following relations $[20,27,32]$ :

$n\left(V_{i}\right)=\frac{q}{k T}\left[\frac{V_{i}}{\ln \left(\frac{I_{i}}{I_{0}}\right)}\right]=1+\frac{\ddot{a}}{\varepsilon_{i}}\left[\frac{\varepsilon_{s}}{W_{D}}+q N_{s s}\left(V_{i}\right)\right]$
$\hat{\mathrm{O}}_{e}=\hat{\mathrm{O}}_{B 0}+\hat{a}(V)=\hat{\mathrm{O}}_{B 0}+\left[1-\frac{1}{\mathrm{n}\left(\left(V_{i}\right)\right.}\right] V_{i}$

where $\beta$ is the voltage coefficient of the effective $\mathrm{BH}$ used in the $\Phi_{B O}\left(B=d \Phi_{e} / d V=1-1 / n(V)\right)$. In calculations, the value of $\delta$ is taken as $20 \AA$ for native $\mathrm{SiO}_{2}$ and $500 \AA$ for (Zn-doped PVA) layer respectively. $W_{D}$ is the width of the space charge region $(\approx 3.87 \times$ $10^{-5} \mathrm{~cm}$ which can be calculated from the linear part of reverse bias $C^{-2}$ vs. $V$ plot at $1 \mathrm{MHz}$ ). The value of $\varepsilon_{s}$ was taken as $13.1 \varepsilon_{o}$ for $4 \mathrm{H} \mathrm{SiC}$, the values of $\varepsilon_{i}$ was taken as $3.8 \varepsilon_{o}$ for $\mathrm{SiO}_{2}$ and $8.47 \varepsilon_{o}$ for $\mathrm{Zn}$-doped PVA. The voltage dependent $n\left(V_{i}\right)$ for both structures at room temperature are obtained by using eqn. (4) and shown in
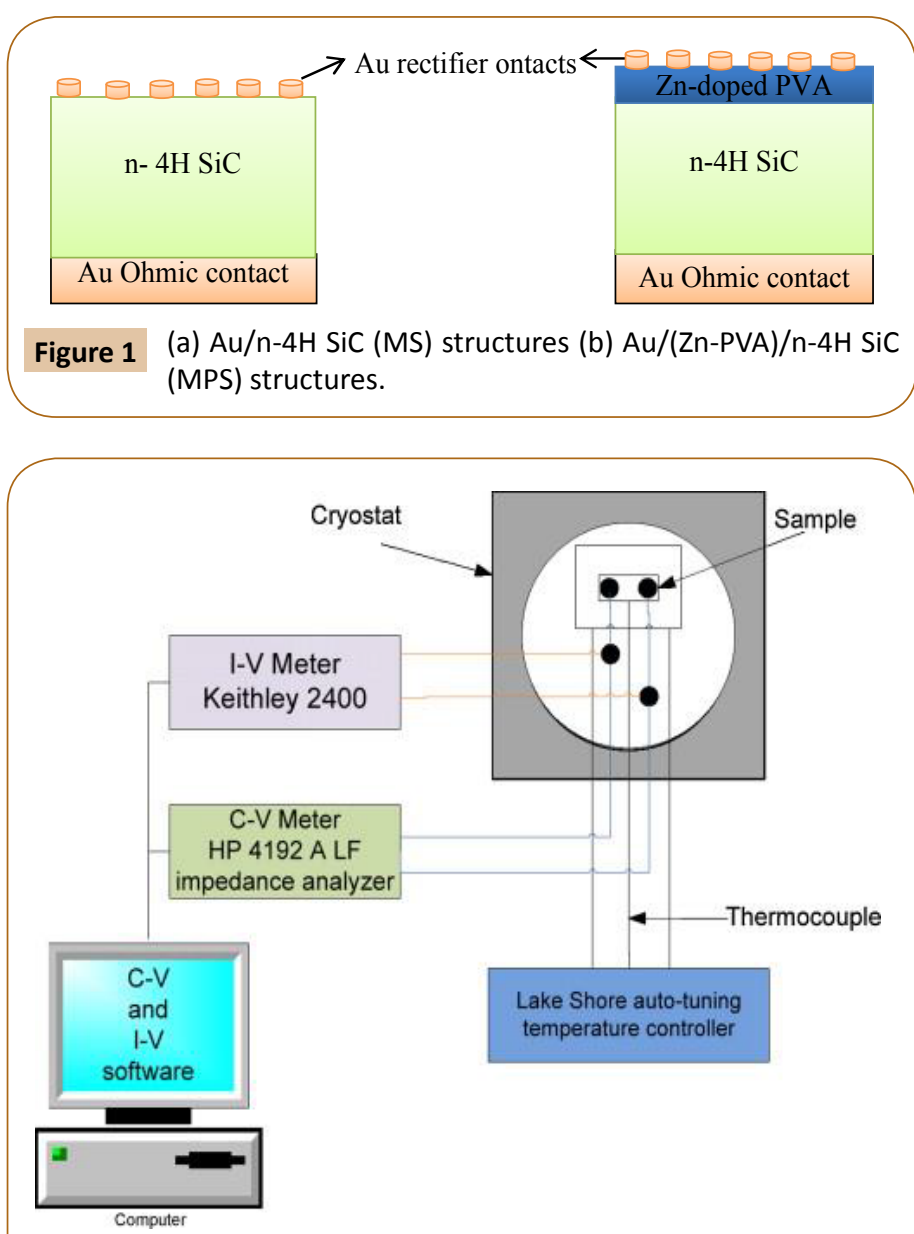

Figure 2 A simple illustration of electrical measurements system.

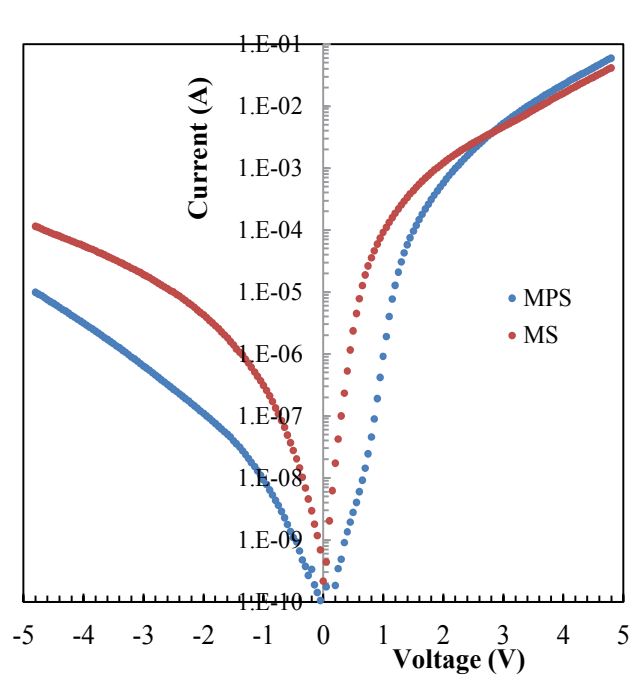

Figure 3 Experimental reverse and forward bias $I-V$ characteristics for both MS and MPS type structures at room temperature. 
Table 1 The experimental results values for some electrical parameters obtaind from the forward bias $I-V$ characteristics for MS and MPS structure at room temperature.

\begin{tabular}{|c|c|c|c|c|c|c|c|c|}
\hline Samp. & $I_{0}(A)$ & $n(I-V)$ & $\Phi_{B 0}(I-V)(e V)$ & $R_{s}(d V / d \ln (l)-I)(\Omega)$ & $R_{s}(H(I)-I)(\Omega)$ & $R R( \pm 6 V)\left(=I_{F} / I_{R}\right)$ & $R_{s}(I-V)(\Omega)$ & $R_{s h}(I-V)(\Omega)$ \\
\hline MS & $2.91 \times 10^{-11}$ & 3.854 & 0.806 & 27.03 & 89.305 & 347 & 49.4 & $2.4 \times 10^{7}$ \\
\hline MPS & $7.08 \times 10^{-10}$ & 3.241 & 0.815 & 171.72 & 196.79 & 5390 & 22.1 & $9.5 \times 10^{8}$ \\
\hline
\end{tabular}

Figure 4. the experimental results value of $\mathrm{n}$ at intermediate bias voltage (for the linear region of Ln/ vs. $V$ plots) is almost constant, but it becomes increase with increasing voltage almost linearly at enough high forward bias voltages for two diodes.

In addition, the expression for the $N_{s s}$ that is entirely governed by the semiconductor suggested by Card and Rhoderick can be simplified as follows [32].

$$
N_{s s}(V)=\frac{1}{q}\left[\frac{\varepsilon_{i}}{\delta}(n(V)-1)-\frac{\varepsilon_{s}}{W_{D}}\right]
$$

The energy level of $N_{s s}$ with respect to the bottom of the conduction band for $n$-type semiconductor is given by $[7,21]$

$$
E_{c}-E_{s s}=q\left(\hat{\mathrm{O}}_{e}-V\right)
$$

From eqn. (4), it's clear that the value of $\Phi$ increases by increasing applied forward bias voltage because of the increase in quasiFermi energy level of majority carriers on the semiconductor side. Thus, the value of $N_{s s}$ was calculated by using eqn. 5(a) and 5(b) and was given in Figure $\mathbf{5}$ for both structures. As can be seen clear in Figure 5, the values of $N_{s s}$ increase from the mid-gap of $4 \mathrm{H}$ $\mathrm{SiC}$ towards the bottom of conduction band $\left(E_{c}\right)$. Thus the values of $N_{s s}$ for the MPS are much lower like $n$ and leakage current compared to the MS because of the saturation of dangling bonds or the passivity role of ( $\mathrm{n}$ doped-PVA) interfacial layer. These results shows a grown of a modified PVA interfacial layer doped by $\mathrm{Zn}$ nanoparticles between metal and semiconductor instead of conventional $\mathrm{SiO}_{2}$ can increases the quality and performance of the MS and MIS type structures (Figure 5).

Usually, the forward bias I-V plot for MS, MIS and MPS structures is linear on semi-logarithmic scale at intermediate forward bias voltage $(V \geq 3 k T / q)$, but there it becomes deviation from linear state especially because of the exist of $R_{s}$ and polymeric interfacial layer. Furthermore, the experimental values of $N_{s s}$ and resistance of the interlayer will be added to the resistance of epitaxial and so leads to deviate from linearity at enough high forward biases [17]. There are several methods to determine the value of $R_{s}$ in the literature [18-20,22-24]. In this study, $R_{s}$ has been calculated by applying the Cheung's method [24]. by using this method, the forward bias I-V relation with $R_{s}$ value can expressed as:

$$
\frac{d V}{d(\ln I)}=n \frac{k T}{q}+I R_{s}
$$

and

$$
H(I)=V-n \frac{k T}{q} \ln \left(\frac{I}{A A^{*} T^{2}}\right)=n \phi_{B}+I R_{s}
$$

Both the $d V / d(\ln /)$ vs. I and $\mathrm{H}(I)$ vs. I plots were drawn by using eqns. (6) and (7) for the MS and MPS structures ant they were given in Figures 6 and 7, respectively. As shown in Figures 6 and
7, these two plots have a good linear range for two structures. Thus, a plot of $\mathrm{dv} / \mathrm{dln}$ I vs. I will give the values of $R_{s}$ as the slope and $n$ values which determined from eqn. (6) which represent the $\mathrm{y}$-axis intercept $(=n k T / q)$, of these plots respectively. Similarly, the slope of $\mathrm{H}(I)$ vs. I plot (Figure 7) will provide a second way for determination of $R_{s}$, which produce a check the uniformity of this approach. From eqn. (7), the values of $R_{S}$ and $\Phi_{B}$ were calculated and obtained from the slope and the intercept of $y$-axis $\left(=n \Phi_{B}\right)$ of these plots for two structures, respectively. The values of $n$ which are calculated from Cheung function acorrding to eqn. (6) are considerably higher than those extractes from eqn. (2). Figure 4, shows an expected situation due to the increase of $n$ with applied bias voltage. The voltage dependence of structure resistance $\left(R_{i}\right)$ is also obtained by applying Ohm's law $\left(R_{i}=d V_{i} / d I_{i}\right)$ and represented inset in Figure $\mathbf{3}$ for MS and MPS type structure. As shown in this figure, the real values of $R_{s}$ and $R_{s h}$ for both two structures agree to enough high forward bias voltage (6V) and enough low or zero bias voltages, respectively. As seen in Table 1 , the experimental result of $R_{s h}$ value for MPS structure is 39.99 times higher as compare with $R_{s h}$ value for MS structure. The experimental values of $R_{s}$ that obtained from Cheung's functions and Ohm's law for two structures were represented in Table 1. The discrepancy between $R_{s}$ from these methods is the result of voltage dependence of it like ideality factor and $\mathrm{BH}$.

\section{The forward and reverse bias $C-V$ and $G / \omega-V$ characteristics}

The capacitance and conductance measurements ( $C-V$ and $\mathrm{G} / \omega-V)$ respectively were applied at both low $(1 \mathrm{kHz})$ and high (1 MHz) frequencies by using HP 4192A LF Impedance Analyzer. According to Nicollian and Brews [22], at enough low frequencies, almost of all surface states can follow the external alternating ac signal easily and so yield an excess $C_{\text {ex. }}$ and $G_{e x} / \omega$ to the real values of them, but at high frequencies these states/traps impossible follow the ac signal. For this purpose, the $C-V$ and $G / \omega-V$ plots of the MS and MPS structures were drawn at low frequency 1 $\mathrm{kHz}$ and high frequency $1 \mathrm{MHz}$. Figure $8 \mathrm{a}$ and $8 \mathrm{~b}$ shows the $\mathrm{C}-\mathrm{V}$ characteristics for MS and MPS structures at $1 \mathrm{kHz}$ and $1 \mathrm{MHz}$, respectively, and these plots have three regions (inversion, depletion and accumulation) both low and high frequency. $C-V$ characteristic has shown clearly that the anomalous peak at the accumulation region due to the effect of $R_{s}$ and native $\mathrm{SiO}_{2}$ and (Zn-doped PVA) interfacial layer rather than $N_{s s}$. It is clear that the magnitude of anomalous peak inversely related to frequency due to the effect of $N_{s s}$. This kind of peak behavior can be attributed to the particular density distribution of $N_{s s}$ and remodeling and restructure of them under applying electric field [32-34]. As seen in Figure $\mathbf{8 a}$ and $\mathbf{8 b}$ the value of capacitance of both structures are extremely sensitive to the $N_{s s}$ interfacial layer and $R_{s}$, but $N_{s s}$ shows an effective behavior especially at inversion and 
depletion regions, whereas $R_{s}$ shows an effective behavior only at accumulation region.

As seen in Figure $\mathbf{8 b}$, at $1 \mathrm{MHz}, \mathrm{C}$ value increases with increasing voltage until accumulation region after that it suddenly decrease until goes to negative due to the effect of $R_{s}$, but at $1 \mathrm{kHz}$, this negative capacitance (NC) behavior becomes disappear. Because, the effect of $R_{s}$ on $C-V$ and $G / \omega$-V plots can be neglected low at low frequencies, but it becomes very effective at high frequencies contrary to $N_{s s}$. The general consensus on NC in the accumulation region is due to the existence of $N_{s s}$ and their relaxation time $(\tau)$, $R_{s}$, the contact injection and minority carrier injection $[35,36]$. In

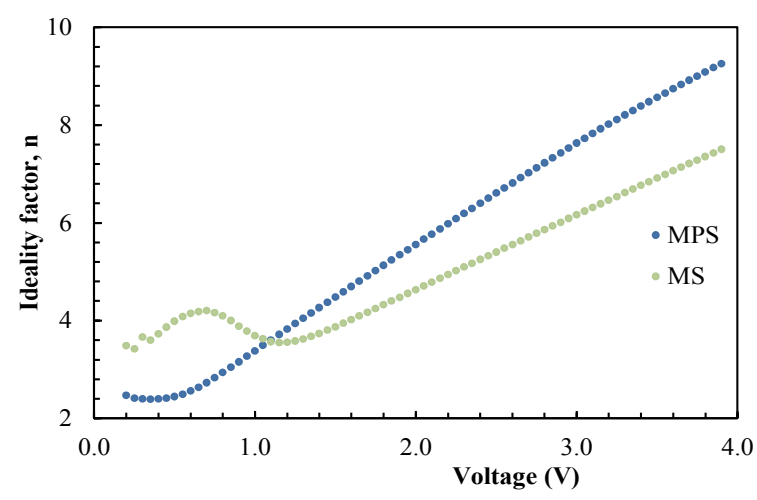

Figure $4 n$ vs. $V$ for both MS and MPS structures at room temperature.

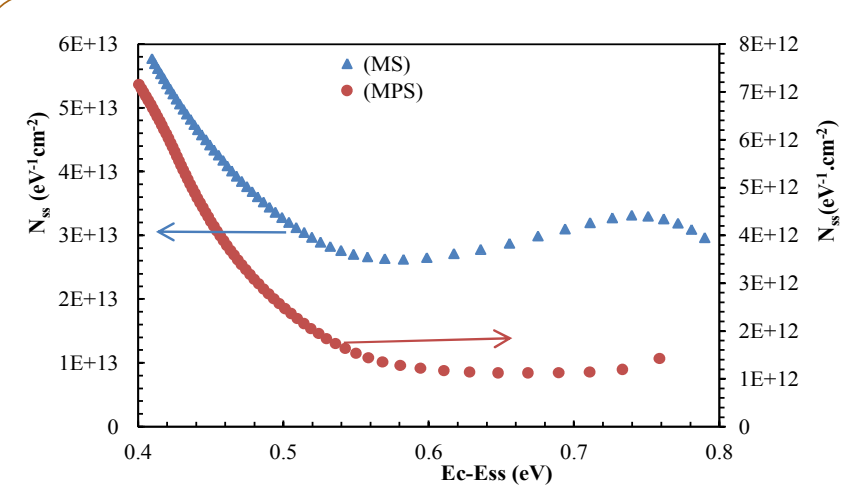

Figure 5 The energy dependent density distribution profiles of $N_{s s}$ vs. $\left(E_{c}-E_{s s}\right)$ plots for the MS and MPS structures at room temperature.
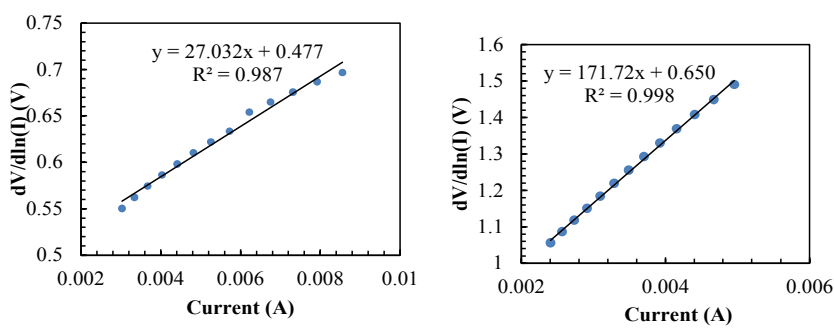

Figure 6

(a) $d V / d \ln (I)$ vs. I for MS structure. (b) $d V / d \ln (I)$ vs. I for MPS structure. addition, according to Butcher et al. [37] and Huang et al. [38], NC behavior can be attributed to technical problems of devices, such as parasitic inductance or accuracy of calibration, respectively (Figure 8).

In general, the NC has been referred to as "abnormal" or "anomalous" behavior in the literature [33]. The $C$ value is a differential effect of charge $Q$ with respect to junction voltage $V_{j}$, i.e. $C=d Q / d V_{j}$ Zhu et al. [39]. Furthermore, by increasing forward bias voltage the $C$ values reach to a maximum value and after certain voltage it started rapidly in decreasing until reach to negative value $[35,39,40] . C-V$ and $G / \omega-V$ plots for MS and MPS structures at $1 \mathrm{MHz}$ were drawn to see the inductive behavior in these structures in Figure 9a and 9b, respectively, which explain how the $G / \omega$ value increases for two structures with increasing frequency both at depletion and accumulation region, but at accumulation region contrary to $G / \omega$, the value of $C$ reaches a peak value and then goes to negative value at strong accumulation region. In addition, the minimum value of $C$ represent the maximum value of $G / \omega$. NC exhibits a decrease in electronic charges on electrodes with an increasing bias voltage and such behavior of $C$ and $G / \omega$ is known as "inductive behavior" in the literature [33-40] (Figure 9).

The variation in $C$ at the reverse bias or inversion region, the $C^{-2}-V$ plot of the MS and MPS structures for $1 \mathrm{MHz}$ are given in Figure 10. As shown in Figure 10, the $C^{2}-V$ plot for two different type structures has a perfect linear region in the wide range of reverse bias voltage. At elevated frequencies $(f \geq 1 \mathrm{MHz})$, the depletion layer capacitance of MS and MPS type structures can be expressed as following [18-20].
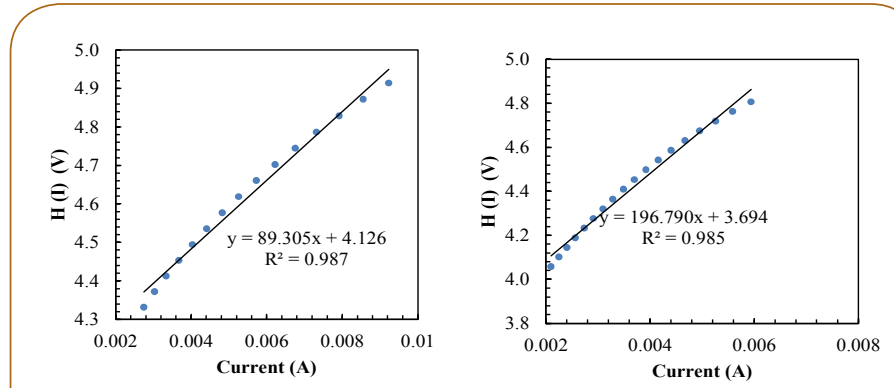

Figure 7 (a) $H(I)$ vs. I for MS structure. (b) $H(I)$ vs. I for MPS structure.
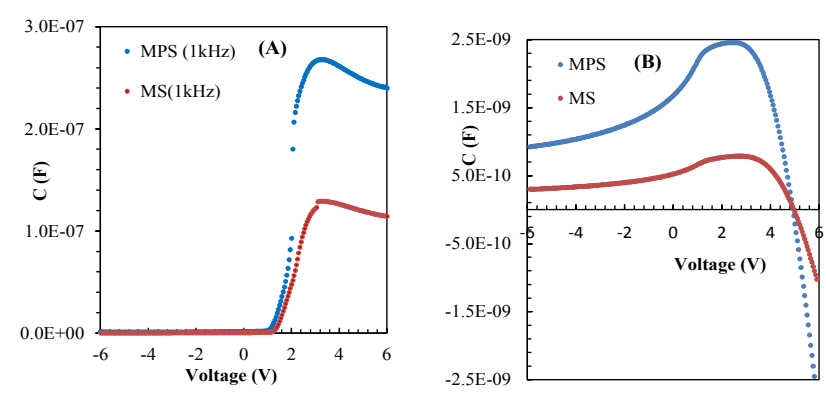

Figure 8 (a) $C-V$ characteristics of MS and MPS at $1 \mathrm{kHz}$ (b) $C-V$ characteristics of MS and MPS at $1 \mathrm{MHz}$. 

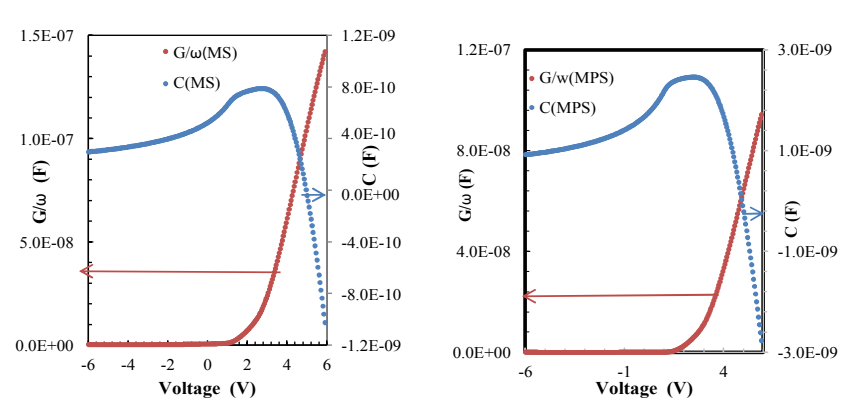

Figure 9 (a) $C-V$ and $G / \omega-V$ plots of $M S$ at $1 \mathrm{MHz}$. (b) $C-V$ and $G / \omega$ characteristics of MPS at $1 \mathrm{MHz}$.

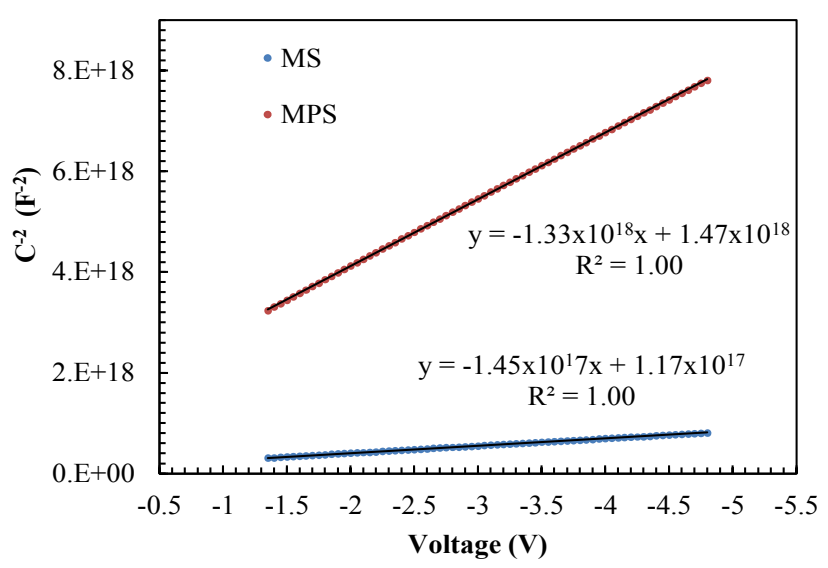

Figure $10 \mathrm{C}^{-2}-\mathrm{V}$ plots for MS and MPS structures for $1 \mathrm{MHz}$ at room temperature.

$C=\frac{\left|d Q_{s c}\right|}{|d V|}=\sqrt{\frac{q \varepsilon_{\mathrm{r}} \varepsilon_{0} A^{2} N_{D}}{2\left(V_{b i}+V\right)}}$ and $\frac{1}{C^{2}}=\frac{2\left(V_{R}+V_{b i}\right)}{q \varepsilon_{r} \varepsilon_{0} N_{d} A^{2}}$

In eqn. (8), $A$ represent the rectification contact area, $V_{R}$ represent the reverse-bias voltage, $V_{b i}$ represent the built-in voltage at zero bias, $N_{d}$ represent the donor concentration atoms in $4 \mathrm{H} \mathrm{SiC}, \varepsilon_{r}$ represent the permittivity of semiconductor and $\varepsilon_{0}$ represent the permittivity of free space or vacuum. The values of $V_{o^{\prime}} N_{d^{\prime}} E_{F^{\prime}}$ $\Phi_{b}(C-V)$ and depletion layer width $\left(W_{D}\right)$ are calculated from the intercepts and slopes of the $C^{2}-V$ plot for each structure using following relations [18-21]:

$V_{b i}=V_{0}+\frac{k T}{q}$

$W_{D}=\left[\frac{2 \varepsilon_{r} \varepsilon_{0}\left(V_{R}+V_{b i}\right)}{q N_{d}}\right]^{\frac{1}{2}}$

The value of $E_{F}$ can be calculated from the conduction band edge for $n$-type semiconductor in the neutral region of $n-4 \mathrm{H}$ SiC by using following relation as $[18,19]$ :

$E_{F}=\frac{k T}{q} \ln \left(\frac{N_{c}}{N_{d}}\right)$

Where, $N_{c}$ is the effective density of states in $\mathrm{n}-4 \mathrm{H}$ SiC conduction band. The experimental values $V_{o^{\prime}} N_{d^{\prime}} E_{F^{\prime}} \Phi_{B}$ and $W_{D}$ were obtained and arranged as in Table $\mathbf{2}$.
As can be seen in Tables 1 and 2, the $\Phi_{B O}(C-V)$ value which calculated from the reverse bias $C-V$ characteristics is higher than $\Phi_{B O}(I-V)$ which calculated from the forward bias $I-V$ characteristics due to the nature of measurement methods. In other words, the values of $\mathrm{BH}$ obtained from two techniques are not always the same in bias voltage. It is well known that the apparent $\mathrm{BH}$ from metal side to semiconductor side is higher than at about $E_{F}$ from semiconductor to metal sides. This discrepancy between $\Phi_{B O}$ $(C-V)$ and $\Phi_{B O}(I-V)$ is also the result of the in-homogeneities of $\mathrm{BH}$ and polymeric interfacial layer at $\mathrm{M} / \mathrm{S}$ interface.

The profile of $R_{s}$ as voltage dependent was also calculated by using eqn. (12) for the $C-V$ and $G / \omega$ measurements by applying Nicollian and Brews method at $1 \mathrm{MHz}$ to explain how $R_{s}$ effects on both $C-V$ and $G / \omega-V$ characteristics for MS and MPS structure and were given in Figure 11 [22].

$$
R_{s}=\frac{G_{m i}}{G_{m i}^{2}+\left(\omega C_{m i}\right)^{2}}
$$

As shown in Figure 11, the value of $R_{s}$ at accumulation region is almost independent from applied bias voltage and so it is representing to the real value of $R_{s}$ for two type structures. Thus, the real value of $R_{s}$ for MS and MPS structure was found as $1.09 \Omega$ and $1.63 \Omega$ at $6 \mathrm{~V}$, respectively. The value of $R_{s}$ for MPS structure is also lower than the MS structure and these values of $R_{s}$ are more suitable for an electronic device. The peak behavior in $R_{s}$ vs. $V$ plot for MPS structure can be caused by particular distribution of $N_{s s}$ at (Zn doped-PVA)/n-4H SiC interface. Because, at peak position, the charge carriers may be trapped due to higher value of $N_{s s}$ and at certain high forward biases voltage the value of $R_{s}$ will decrease due to decreasing of charge carriers at traps or states. Finally, in order to see the charges at surface states or traps on the $C-V$ and $G / \omega-V$ plots, the profiles of $N_{s s}$ vs. $V$ was also calculated from the high-low frequency $(1 \mathrm{MHz}-1 \mathrm{kHz})$ capacitance $\left(\mathrm{C}_{\mathrm{HF}}-\mathrm{C}_{\mathrm{LF}}\right)$ technique using following relation for both structures and they were given in Figure 12.

$q A N_{S S}=\left(1 / C_{L F}-1 / C_{i}\right)^{-1}-\left(1 / C_{H F}-1 / C_{i}\right)^{-1}$

In eqn. (13), $C_{i}$ is the interfacial layer capacitance and was obtained by using the $C_{m a}$ and $G_{m a} / \omega$ values at strong accumulation region at $1 \mathrm{MH}$ by using following relation [22].

$C_{i}=C_{m a}\left[1+\left(\left(G_{m a} / \omega\right) / C_{m a}\right)^{2}\right]$

As seen in Figure 12, the density of $N_{s s}$ for MS and MPS type structures shows a peak value due to a special density distribution of $N_{s s}$ and their remodeling and restructure under electric field. As a result, the values of $N_{s s}$ obtained high-lowfrequency capacitance method for MPS structure are also lower than MS structure at accumulation region, but the distribution of $N_{s s}$ varied from region to region due to their special distribution between interfacial layer and semiconductor. Furthermore,

Table 2 The expermental values of some parameters at the linear parts of the reverse bias $C^{-2}$ vs. $V$ plots for MS and MPS structure at room temperature.

\begin{tabular}{c|c|c|c|c|c|}
\hline Sample & $V_{o}(\mathrm{~V})$ & $N_{d}\left(\mathrm{~cm}^{-3}\right)$ & $E_{F}(\mathrm{eV})$ & $\boldsymbol{\Phi}_{B}(\mathrm{eV})$ & $W_{D}(\mathrm{~cm})$ \\
\hline MS & 0.807 & $8.373 \times 10^{16}$ & 0.123 & 0.955 & $1.249 \times 10^{-5}$ \\
\hline MPS & 1.105 & $9.128 \times 10^{15}$ & 0.179 & 1.309 & $3.781 \times 10^{-5}$ \\
\hline
\end{tabular}




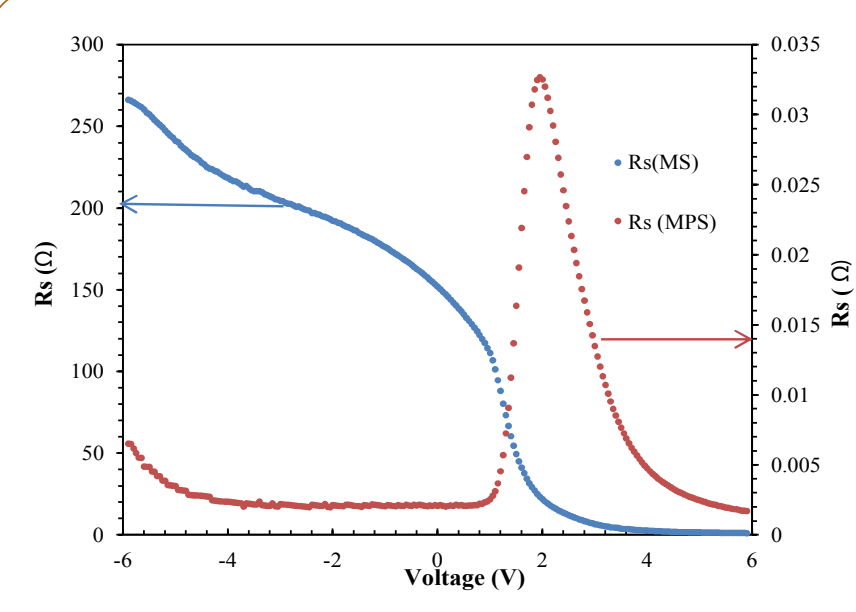

Figure $11 R_{s}$ vs. $V$ plots of the MS and MPS structures for $1 \mathrm{MHz}$ at room temperature.

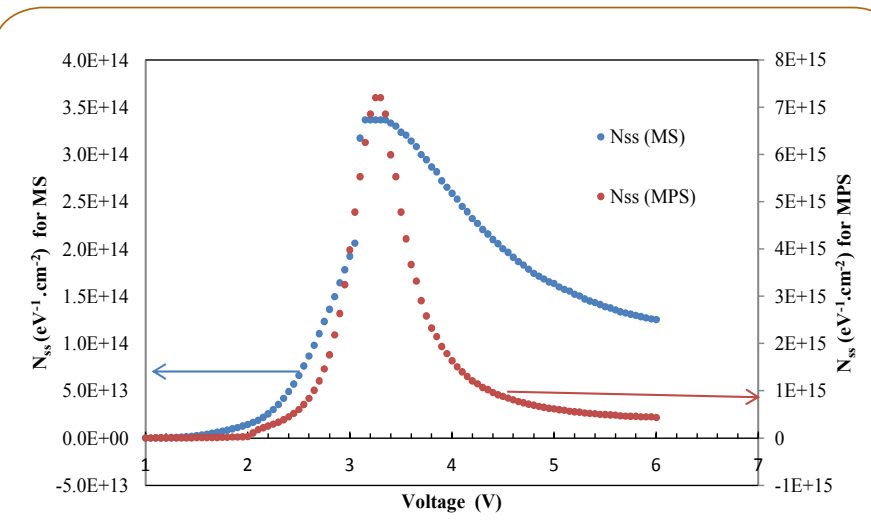

Figure 12

Profiles of $N_{s s}$ vs. $V$ for the MS and MPS structures obtained by the high-low frequency capacitance method at room temperature.

Figure 12 show a peak value of $N_{s s}$ at about $3 \mathrm{~V}$ for both MS and MPS structures with higher value for MPS 10 times than MS structure and then at higher voltage $V \geq 3$ the value of $N_{s s}$ exponentially decreases from conduction band to mid-gap with increasing applied biasing voltage in different ratio for both structures. These results indicated the electrical parameters of these structures to be quite dependent on voltage as well as interfacial polymeric layer. When the main electrical parameters of MS and MPS structure are compared; the values of ideality factor, leakage current, $R_{s}, \Phi_{B o}$ and $N_{s s}$ for MPS structure are considerably lower than the MS structure. However, the values of $R_{s h}$ and $R R$ for MPS structure are considerably higher than the MS structure due to the passivity role of Zn-PVA interfacial polymer layer. As a result, we can say that the ( $Z n$ doped-PVA) polymer material can be applied as an interfacial layer instead classical $\mathrm{SiO}_{2}$ insulation layer to get better quality or performance of MS structure.

\section{Conclusion}

In this study, how the effect of modified PVA interfacial layer doped by $\mathrm{Zn}$ nanoparticles on the electrical parameters was evaluated and investigate after fabrication of both $\mathrm{Au} / \mathrm{n}-4 \mathrm{H} \mathrm{SiC}$ (MS) and Au/(Zn-doped PVA)/n-4H SiC (MPS) structures with the same substrate $-4 \mathrm{H} \mathrm{SiC}$ wafer. The main important electrical parameters are obtained from forward and reverse $I-V, C-V$ and $G / \omega-V$ characteristics at room temperature to compare of them. These results show that the main electrical parameters of these MS and MPS structures are considerably dependent on voltage as well as interfacial layer.

the comparison between the two structures showing the that the higher values of $\mathrm{BH}$ obtained from the reverse bias $C-V$ data as compare with the forward bias $I-V$ data for the two structures due to the nature of the used of measurement technique. The high values of $\mathrm{n}$ for two structures were caused by the existence of the native and doped interfacial layer, lower barriers or patches at around mean value of $\mathrm{BH}$ and the existence of $N_{s s}$ at interfacial layer/n-4H SiC interface. The profiles of $N_{s s}$ vs. $V$ was measured by using the forward bias $I-V$ data by taking into account the voltage dependent $\mathrm{BH}$ and $\mathrm{n}$ for these two structures. The values of $N_{s s}$ increase an exponential from the mid-gap of $\mathrm{n}-4 \mathrm{H} \mathrm{SiC}$ toward the bottom of its $E_{c}$. On the other hand, the profile of $R_{s}$ for these structures was measured from the $C-V$ and $G / \omega-V$ data by applying Nicollian and Brews method. These obtained experimental results are confirmed that the effect of presence of $N_{s s}, R_{s}$, and the interfacial layer is most influential on the electrical parameters of the MS and MPS structures. In conclusion, when the main electrical parameters of both structures are compared; the values of $n$, leakage current, $R_{s}, \Phi_{B o}$ and $N_{s s}$ for MPS structure are lower than the MS structure, but the values of $R_{s h}$ and $R R$ for MPS structure are higher than the MS structure due to the passivity role of Zn-PVA interfacial polymer layer. As a result, we can say that the ( $Z n$ doped-PVA) polymer material when applied as interfacial layer instead of classical $\mathrm{SiO}_{2}$ insulator layer, this will improve the quality or performance of MS structure.

\section{Acknowledgments}

This study is corroborative by Süleyman Demirel University (project no: 4614-D2-16). 


\section{References}

1 Reddy MSP, Kang HS, Lie JH, Reddy VR, Ja Jang S (2014 Electrical Properties and the Role of inhomogeneities at the Polyvinyl Alcohol/ n-InP Schottky Barrier Interface. Appl Polym Sci 131: 139773-10.

2 Gülü Ö, Aydoğan Ş, Türüt A (2012) Electronic parameters of high barrier Au/Rhodamine-101/n-Inp Schottky diode with organic interlayer. Thin Solid Films 520: 1944-1948.

3 Altındal Yeriskin S, Balbaşı AM (2016) Tataroğlu, Frequency and voltage dependence of dielectric properties, complex electric modulus, and electrical conductivity in $\mathrm{Au} / 7 \%$ graphene dopedPVA/n-Si (MPS) structures. J Appl Polym Sci 133: 43827.

4 Reddy VR (2014) Electrical properties of Au/polyvinylidene fluoride/ n-InP Schottky diode with polymer interlayer. Thin Solid Films 556: 300.

5 Taşçıoğlu i, Tüzün Özmen Ö, Sağban HM, Yağlıoğlu E, Altındal Ş (2017) Frequency Dependent Electrical and Dielectric Properties of Au/P3HT: PCBM: F4-TCNO/n-Si Schottky Barrier Diode. J Electron Mater 46: 2379-2386.

6 Özdemir AF, Aydın SG, Aldemir DA, Görsoy SS (2011) Electrical and optical properties of $p$-type silicon based on polypyrrole-derivative polymer. Synth Met 161: 692-697.

7 Altındal S, Sarı B, Ünal HI, Yavas N (2009) Electrical Characteristics of Al/Polyindole Schottky Barrier Diodes. I. Temperature Dependence. J Appl Polym Sci 113: 2955-2961.

8 Bilkan Ç, Zeyrek S, San SE, Altındal Ş (2015) A compare of electrical characteristics in $\mathrm{Al} / \mathrm{p}-\mathrm{Si}$ (MS) and $\mathrm{Al} / \mathrm{C} 2 \mathrm{OH} 12 / \mathrm{p}-\mathrm{Si}$ (MPS) type diodes using current-voltage (I-V) and capacitance-voltage (C-V) measurements. Mater Sci and Semicon Process 32: 137-144.

9 Moraki K, Bengi S, Zeyrek S, Bülbül MM, Altnndal Ş (2016) Temperature dependence of characteristic parameters of the $\mathrm{Au} /$ $\mathrm{C} 2 \mathrm{OH} 12 / \mathrm{n}$-Si Schottky barrier diodes (SBDs) in the wide temperature range. J Mater Sci: Mater Electron 28: 3987-3996.

10 Çicek Ö, Uslu Tecimer H, Tan SO, Tecimer H, Altındal Ş, et al. (2016) Evaluation of electrical and photovoltaic behaviours as comparative of $\mathrm{Au} / \mathrm{n}-\mathrm{GaAs}(\mathrm{MS})$ diodes with and without pure and graphene (Gr)-doped polyvinyl alcohol (PVA) interfacial layer under dark and illuminated conditions. Composites Part B 98: 260.

11 Demir A,Yücedağ i, Ersöz G, Altındal Ş, Baraz N, Kandaz M (2016) A Comparative Study on the Main Electrical Parameters of $\mathrm{Au} / \mathrm{n}-\mathrm{Si}, \mathrm{Au} /$ Biphenyl-CuPc/n-Si/and Au/Biphenylsubs-CoPc/n-Si/Type Schottky Barrier Diodes. J. Nanoelectron and Optoelectron 11: 620-625.

12 Bülbül MM, Bengi S, Dökme I, Altındal Ş, Tunç T (2010) Temperature dependent capacitance and conductance-voltage characteristics of $\mathrm{Au} /$ polyvinyl alcohol(Co,Zn)/n-Si Schottky diodes, J. Appl. Phys., 108: 34517-1-034517-6.

13 Altındal Ş, Uslu H (2011) The origin of anomalous peak and negative capacitance in the forward bias capacitance-voltage characteristics of Au/PVA/n-Si structures. J Appl Phys 109: 074503-1-074503-7.

14 Tunc T, Altındal Ş, Uslu I, Dökme I (2011) Temperature dependent current-voltage (I-V) characteristics of Au/n-Si (111) Schottky barrier diodes with PVA(Ni,Zn-doped) interfacial layer. Mat Sci Sem Proc 14: 139-145.

15 Gökçen M, Tunç T, Alttndal Ş, Uslu i (2012) Electrical and photocurrent characteristics of Au/PVA (Co-doped)/n-Si photoconductive diodes. Mater Sci and Eng B 177: 416-420.
16 Casady JB, Johnson RW (1996) Status of silicon carbide (SiC) as a wide-bandgap semiconductor for high-temperature applications: A review, Solid State Electron 39: 1409.

17 Sochacki M, Kolendo A, Szmidt J, Werbowy A (2005) Properties of $\mathrm{Pt} / 4 \mathrm{H}-\mathrm{SiC}$ Schottky diodes with interfacial layer at elevated temperatures. Solid State Electron 49: 585.

18 Sze SM (1981) Physics of Semiconductor Devices, John Wiley and Sons, Toronto.

19 Rhoderick EH (1978) Metal-Semiconductor Contacts, Oxford University Press.

20 Sharma BL (1984) Metal-semiconductor Schottky Barrier Junctions and their applications, Plenum Press, New York and London.

21 Card HC, Rhoderick EH (1971) Studies of tunnel MOS diodes I. Interface effects in silicon Schottky diodes. J Phys D-Appl Phys 4 : 1589-1601.

22 Nicollian EH, Brews JR (1982) MOS Physics and Technology, Wiley, New York.

23 Norde H (1979) A modified forward I-V plot for Schottky diodes with high series resistance. J Appl Phys, 50: 5052-5053.

24 Cheung SK, Cheung NV (1986) Extraction of Schottky diode parameters from forward current-voltage characteristics Appl Phys Lett 49: 85-87.

25 Tung RT (2001) Recent advances in Schottky barrier concepts. Mat Sci Engineerring 35: 1.

26 Werner JH, Guttler H (1991) Barrier inhomogeneities at Schottky contacts. J Appl Phys, 69: 1522-1533.

27 Alialy S, Altındal Ş, Tanrıkulu EE, Yıldız DE (2014) Analysis of temperature dependent current-conduction mechanisms in $\mathrm{Au} /$ $\mathrm{TiO} / \mathrm{n}-4 \mathrm{H}-\mathrm{SiC}$ (metal/insulator/semiconductor) type Schottky barrier diodes. J Appl Phys, 116: 083709.

28 Kimoto T, Cooper JA (2014) Fundamentals of Silicon Carbide Technology (1.st Edn) 2014 John Wiley \& Sons Singapore.

29 Kamoun O, Boukhachem A, Yumak A, Petkova P, Boubaker K, et al. (2016) Europium incorporation dynamics and some physical investigations within $\mathrm{ZnO}$ sprayed thin films, Materials Science in Semiconductor Processing 43: 8-16.

30 Singh PK, Gaur MS, Chauhan RS (2015) Dielectric properties of solgel synthesized polysulfone-ZnO nanocomposites. J Therm Anal Calorim 122: 725.

31 Demirezen, Kaya A, Vural Ö, Altindal \$̧ (1971) The effect of Modoped PVC+ TCNQ interfacial layer on the electrical properties of $\mathrm{Au} /$ $\mathrm{PVC}+\mathrm{TCNQ} / \mathrm{p}$-Si structures at room temperature, , Materials Sci. in Semicond. Proces., 33 (2015)140-148.32. H.C. Card, E.H. Rhoderick, Studies of tunnel MOS diodes I. Interface effects in silicon Schottky diodes. J Phys D: Appl Phys 4: 1589.

32 Demirezen S, Kaya A, Yerişkin SA, Balbaşı M, Uslu i (2016) Frequency and voltage dependent profile of dielectric properties, electric modulus and ac electrical conductivity in the $\mathrm{PrBaCoO}$ nanofiber capacitors. Results in Phys 6: 180.

33 Arslan E, Bütün S, Şafak Y, Uslu H, Taşçıoğlu I, et al. (2011) Electrical characterization of MS and MIS structures on AIGaN/AIN/GaN heterostructures, Microelectron. Rel 51: 370.

34 Uslu H, Altındal Ş, Tunç T, Uslu I, Mammadov TS (2011) The illumination intensity and applied bias voltage on dielectric 
properties of au/polyvinyl alcohol (Co, Zn-doped)/n-Si Schottky barrier diodes, J. of Appl Polym Sci 120: 322.

35 Ershov N, Buchanan M, Wasilewski ZR, Jonscher AK (1998) Negative capacitance effect in semiconductor devices. IEEE Transactions on Electrical Devices 45: 2196.

36 Werner J, Levi AFJ, Tung RT, Anzlowar M, Pinto M (1988) Origin of the excess capacitance at intimate Schottky contacts Phys Rev Lett 60: 53-56.

37 Butcher KSA, Tansley TL, Alexiev D (1996) An instrumental solution to the phenomenon of negative capacitances in semiconductors. Solid-State Electron 39: 333-336.
38 Huang XL, Shin YG, Lim KY, Suh EH, Lee HJ, et al. (1997) Thermally induced capacitance and electric field domains in GaAs/Al0.3Ga0.7As quantum well infrared photo detector. Solid-State Electron 41: 845850.

39 Zhu CY, Feng LF, Wang CD, Cong HX, Zhang GY, et al. (2009) Negative capacitance in light-emitting devices. Solid-State Electron 53: 324 328.

40 Bilkan Ç, Gümüş A, Altındal ş (2015) The source of negative capacitance and anomalous peak in the forward bias capacitancevoltage in $\mathrm{Cr} / \mathrm{p}$-si Schottky barrier diodes. (SBDs) Mater Sci Semi cond Proces 39: 484-4911. 